\title{
Computerized J-H loop tracer for soft magnetic thick films in the audio frequency range
}

\author{
G. Loizos ${ }^{1}$ and D. Niarchos ${ }^{1}$. \\ ${ }^{1}$ NCSR “Demokritos”, Institute of Advanced Materials, Physicochemical Processes, Nanotechnology and Microsystems, \\ Athens, 15310, Greece.
}

\begin{abstract}
A computerized J-H loop tracer for soft magnetic thick films in the audio frequency range is described. It is a system built on a PXI platform combining PXI modules for control signal generation and data acquisition. The physiscal signals are digitized and the respective data strems are processed, presented and recorded in LabVIEW 7.0.
\end{abstract}

\section{Introduction}

There is an interest for the development of high performance nanostructured metallic soft magnetic materials as core inductors for high efficiency DC-DC converters [1], [2] and consequently the development of measurements systems for their characterization. The very small volume of the samples requires special techniques. In these cases closing the magnetic circuit and having flux closure is not required or it is even impossible and open sample measurements are needed [3],[4],[5].

Open sample systems such as Vibrating Sample Magnetometers (VSM), SQUID magnetometers incorporate electromagnets or superconducting coils, the remanent or residual magnetic fields of which are not appropriate to measure the low coercive fields of soft magnetic materials. The magneto-optic Kerr effect (MOKE) technique though very sensitive is not suitable for thick samples because it takes hysteresis measurements only from a thin surface layer (few tens $\mathrm{nm}$ in metals). On the other hand, ac hysteresis loop tracers can be an alternative measuring tool for soft magnetic materials characterization. It can give a satisfactory indication of the coercive field because the widening of the J-H loop area due to the eddy current loss contribution at higher than DC frequencies is not significant especially in resistive soft magnetic materials. This paper describes a computerized J-H loop tracer for the magnetic characterization of soft magnetic strips, ribbons and films with thickness down to a few micrometers in the audio frequency range.

\section{System Description}

The computerized system shown in figures 1, 2and 3 consists of a PXI platform where an arbitrary function generator card and a DAQ card are housed. The generated signal isamplified in a power amplifier and fed to the magnetizing solenoid. The magnetic sample is placed inside the solenoid and a pair of pick-up coils is used for polarization, $J$, sensing and air flux compensation. The magnetic field strength, $H$, proportional to the primary current, $i$, is measured using a shunt resistor. The physical signals are digitized and the respective data streams are processed, presented and recorded in LabVIEW 7.0.

The PXI modular instrumentation platform consists of an ADLINK chassis and controller. It is a scalable, high performance PC based system allowing the data processing to occur in line with the actual data flow.

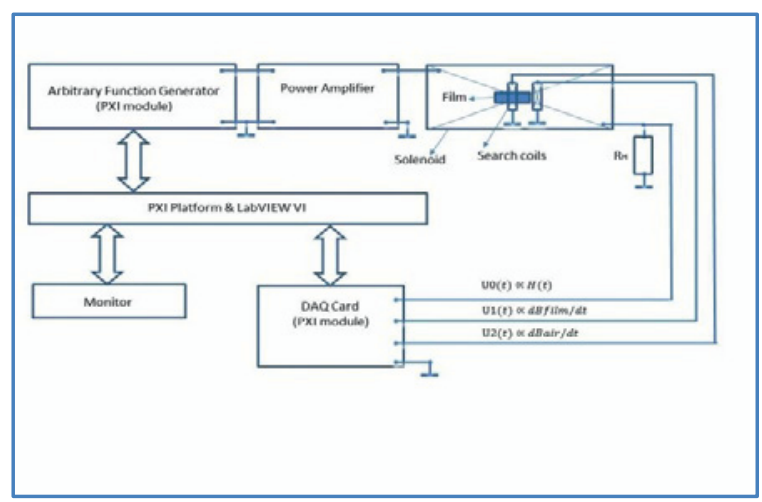

Fig. 1.Basic diagramme of the measurement set-up. 
An arbitrary function generator module, NI PXI-5401 with 12 bit resolution, is selected for signal generation. The signal is fed to a INTER M, R 500 audio frequency power amplifier capable of giving $500 \mathrm{~W}$ into a $8 \mathrm{Ohm}$ load in bridged mono mode or $250 \mathrm{~W}$ into a $4 \mathrm{Ohm}$ in stereo mode.

The solenoid is $230 \mathrm{~mm}$ long, consists of 1000 turns wound in six layers by an enamelled copper wire of 1.18 $\mathrm{mm}$ diameter on a plastic tube with outer diameter 100 $\mathrm{mm}$. The solenoid has also six tappings at 168, 334, 502, 668, 833 and 1000 turns for closer impedance matching with the power amplifier at various frequencies.

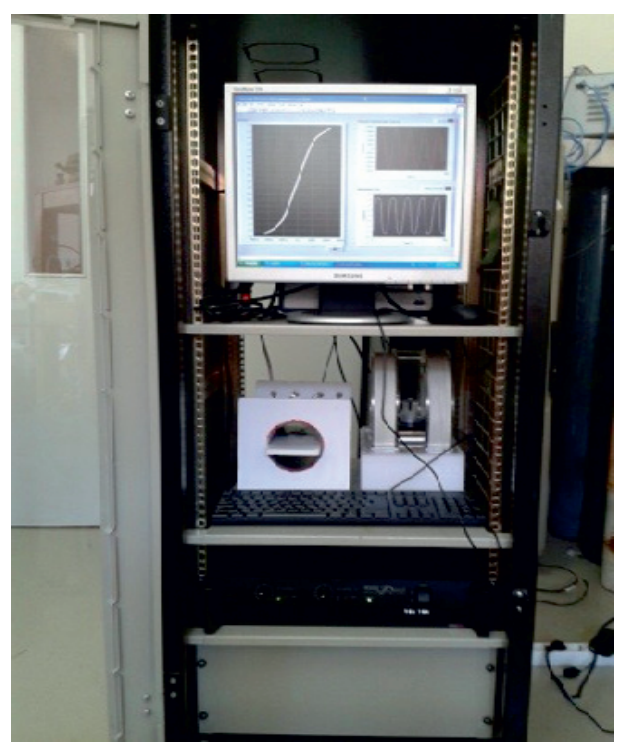

Fig. 2.Photograph of the measurement set-up (front view).

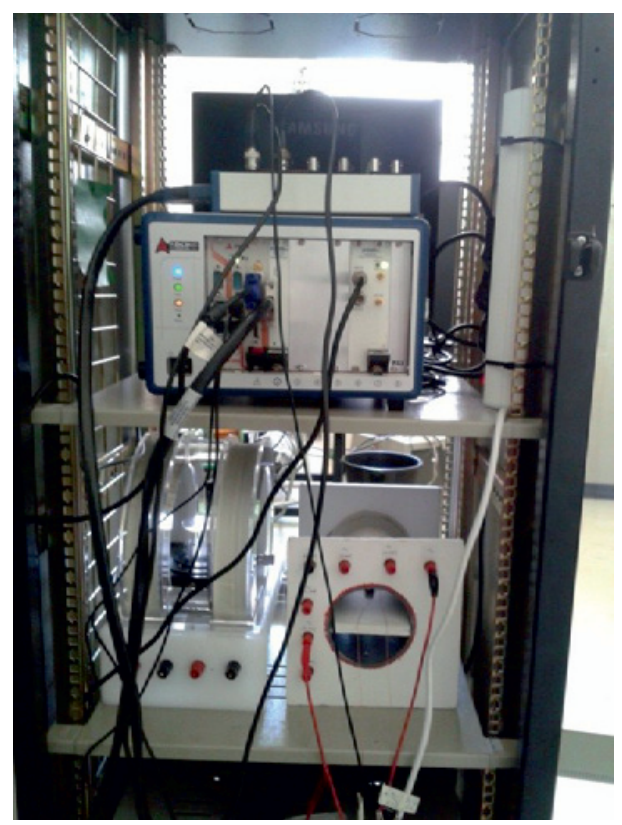

Fig. 3.Photograph of the measurement set-up (rear view).

The maximum magnetic field produced is in the order of $10 \mathrm{kA} / \mathrm{m}$ for twenty minutes.

The samples are placed in the centre of the solenoid for field homogeneity (figure 4). A simple measure to reduce interference from the earth's field in samples is to align them with the east-west direction.

A NI PXI-6251 card with 16 bit resolution, 1MS/s (aggregate) is used to capture the physical voltage signals. This card provides high accuracy at fast sampling rates but not simultaneous sampling a feature necessary for hysteresis measurements. This card presents $1 \mu \mathrm{s}$ interchannel time delay. In this type of system however, where signals are repetitive and low to medium sample rates are required, the hardware simultaneous sampling can be simulated accurately and cost effectively by software waveform-aligning numerical methods, using Align and Resample Express VI provided in LabVIEW 7.0.

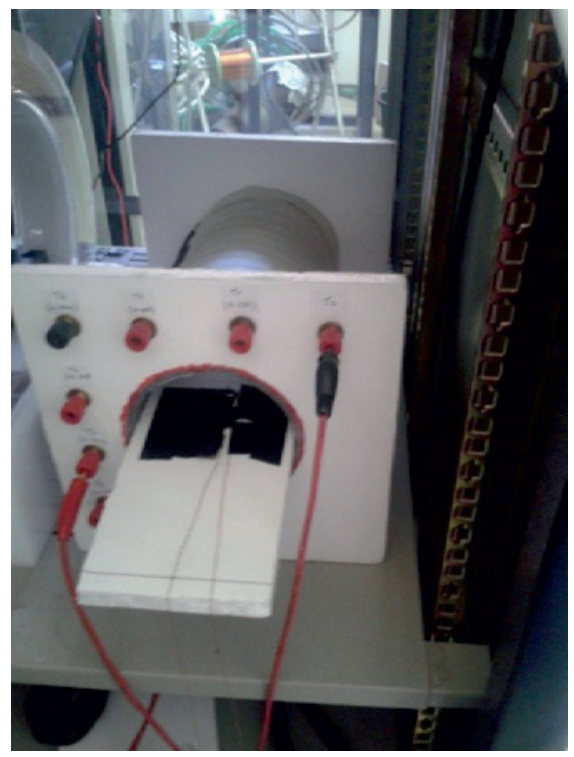

Fig. 4.Solenoid and search coils.

The voltage $U_{0}(t)$ proportional to the magnetic field, $H(t)$, is taken across a low impedance high power resistor $R_{H}$ as shown in Eq. 1 and 2. Eq. 1 relates the magnetic field with the current $i(t)$ in the solenoid of $N$ turns by taking into consideration that it is a thick solenoid with length, $l_{m}$, and outer solenoid diameter, $d$.

$$
\begin{aligned}
& H(t)=\frac{N \cdot i(t)}{l_{m}} \cdot \frac{l_{m}}{\sqrt{l_{m}^{2}+d^{2}}} \\
& i(t)=\frac{U_{0}(t)}{R_{H}}(2)
\end{aligned}
$$

In strips, ribbons and films open samples the air flux contribution cannot be neglected and therefore air flux compensation is needed. A secondary coil and a compensating coil having equal turn-area products were wound one next to the other, leaving a gap between, around a thin non magnetic former. A zero flux variation can be achieved in the absence of the sample if the voltage $U_{2}(t)$ induced in the compensating coil is subtracted in LabVIEW from the voltage $U_{l}(t)$ induced in the secondary coil. In the presence of the sample the resulting linked flux is proportional to the material 
polarization, $J$. No correction for the demagnetizing field has been included.

$U_{1}(t)=N_{2} S_{m} \frac{d J}{d t}+N_{2} S_{2} \cdot \mu_{0} \frac{d H}{d t}$

$U_{2}(t)=N_{2} S_{2} \cdot \mu_{0} \frac{d H}{d t}$

Where $N_{2}$ : number of turns in the secondary and compensating coils, $S_{m}$ : material cross sectional area and $S_{2}$ : total cross sectional area. Air flux compensation is not entirely perfect and it contributes to the uncertainty on $U_{l}(t)$ around $10^{-3}$.

In very small samples the use of a former where the secondary and compensating coils are permanently wound so that the various samples can be inserted for testing does not give satisfactory results. Hence for each type ofsample tested a special former and coils were made ensuring the lowest possible air flux as well as the full flux enclosure of the secondary coil.

After air flux compensation is carried out the resulting stream of data is numerically integrated using the Simpson's rule and magnetic polarization $J(t)$ is obtained. Before integration is performed the residual offsets are numerically eliminated. A trivial but dangerous source of error in $J(t)$ calculation comes from the imperfect determination of the cross sectional area of the specimen. The direct measurement of the strip dimensions does not guarantee a sufficiently accurate determination of $A$. Alternatively the mass $m$ and length $l$ of the test specimen are measured and the cross sectional area is calculated from $A=m / \delta l$, with the density $\delta$ known from the composition or obtained by measurement. It is considered that mass and length of the specimen can be measured with better accuracy and that the major contribution to the uncertainty in the determination of the value of $A$ comes from the measurement of density [6].

The digititized data for the calculated $J(t)$ and $H(t)$ are then presented as $J-H$ hysteresis loop using the appropriate X-Y graph VI in LabVIEW. Data can also be recorded and exported in a txt file format.

\section{Examples of measurements}

Figure 5 shows a hysteresis loop from ribbons of an alloy with nominal composition of $\left(\mathrm{Fe}_{0.5} \mathrm{Co}_{0.5}\right)_{73.5} \mathrm{Si}_{13.5} \mathrm{~B}_{9} \mathrm{Nb}_{3} \mathrm{Cu}_{1}$. The alloy was produced in an arc furnace under argon atmosphere. The rapidly solidified ribbons were melt-spun with a wheel speed of $38 \mathrm{~m} / \mathrm{s}$. The ribbons were sealed in a quartz tube under vacuum and isothermally heat treated at temperature 565 ${ }^{\circ} \mathrm{C}$ for $60 \mathrm{~min}$. The dimensions of each ribbon were $\mathrm{l}=10$ $\mathrm{mm}, \mathrm{w}=1 \mathrm{~mm}$ and $\mathrm{d}=30 \mu \mathrm{m}$.

The measured peak polarization $\mathrm{J}_{\mathrm{p}}$ is at $0.67 \mathrm{~T}$ and the coercive field $\mathrm{H}_{\mathrm{c}}$ is in the order of $20 \mathrm{~A} / \mathrm{m}$. There are not any previously measured data on this material in our possession for comparisons.

Figure 6 shows hysteresis loops from a strip of $2605 \mathrm{HB} 1$ made by Hitachi Metals. Strips dimensions were $1=20$ $\mathrm{mm}, \mathrm{w}=11 \mathrm{~mm}$ and $\mathrm{d}=26 \mu \mathrm{m}$. The measured peak polarization $\mathrm{J}_{\mathrm{p}}$ isat $1.4 \mathrm{~T}$ and the coercive field $\mathrm{H}_{\mathrm{c}}$ is in the order of $30 \mathrm{~A} / \mathrm{m}$ instead of the $1.5 \mathrm{~T}$ and approximately 2 $\mathrm{A} / \mathrm{m}$ given by the manufacturer. Figure 7 shows hysteresis loops from a strip of $\mathrm{Fe}-(3 \% \mathrm{wt}) \mathrm{Si}$ or M130$27 \mathrm{~S}$ grain oriented electrical steel. Strip dimensions were $\mathrm{l}=20 \mathrm{~mm}, \mathrm{w}=9.5 \mathrm{~mm}$ and $\mathrm{d}=0.27 \mathrm{~mm}$.

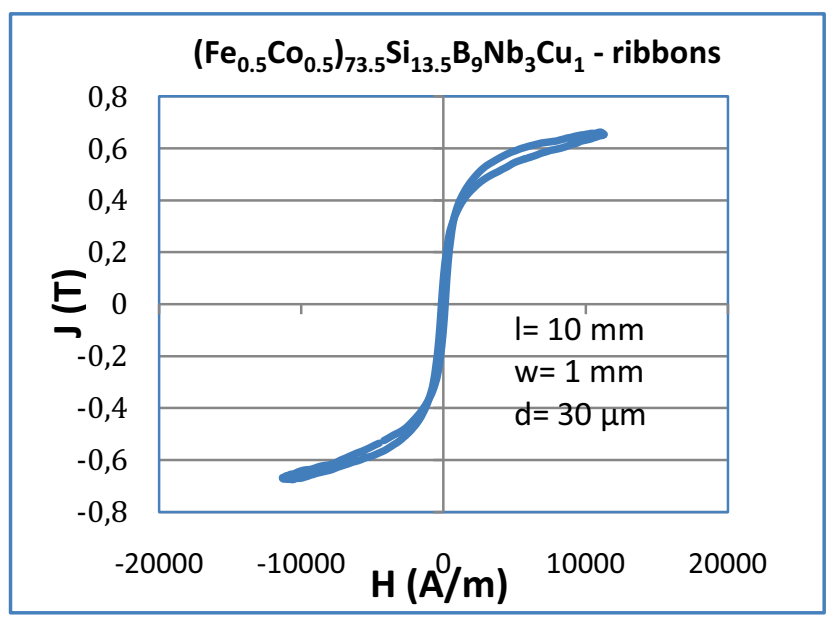

Fig. 5.Hysteresis loop of $\left(\mathrm{Fe}_{0.5} \mathrm{Co}_{0.5}\right)_{73.5} \mathrm{Si}_{13.5} \mathrm{~B}_{9} \mathrm{Nb}_{3} \mathrm{Cu}_{1}$ ribbons magnetized at $50 \mathrm{~Hz}$.

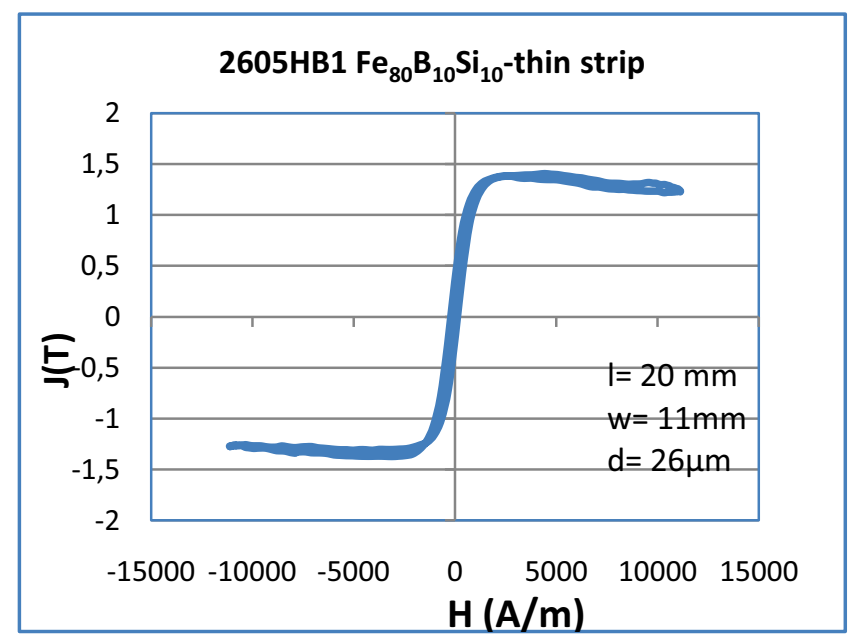

Fig. 6. Hysteresis loop of $2605 \mathrm{HB} 1\left(\mathrm{Fe}_{80} \mathrm{~B}_{10} \mathrm{Si}_{10}\right)$ thin strip magnetized at $50 \mathrm{~Hz}$.

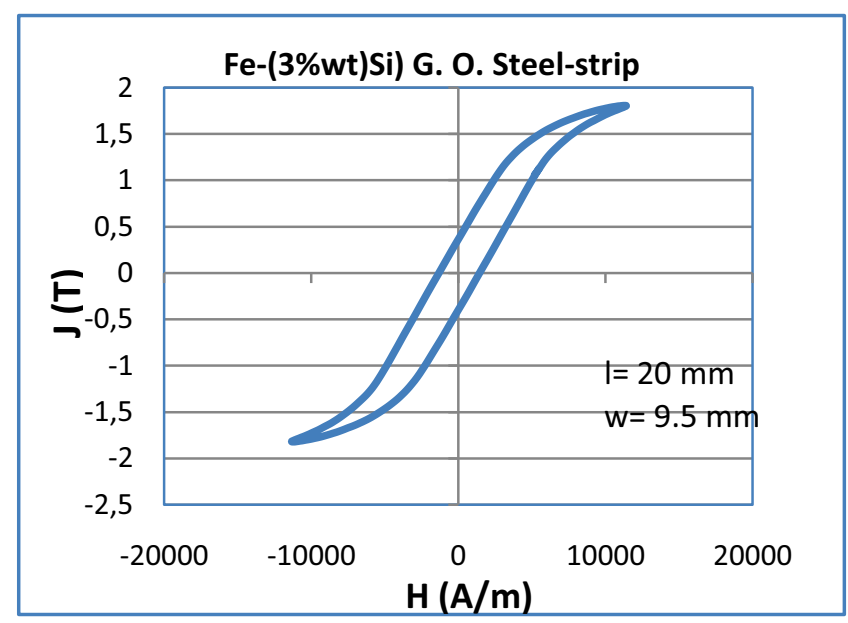


Fig. 7. Hysteresis loop of Fe-(3\%wt)Si G.O. steel strip magnetized at $50 \mathrm{~Hz}$.

The observed peak polarization $\mathrm{J}_{\mathrm{p}}$ is at $1.8 \mathrm{~T}$ and the coercive field $\mathrm{H}_{\mathrm{c}}$ is in the order of $60 \mathrm{~A} / \mathrm{m}$ instead of the 1.85-1.9 $\mathrm{T}$ and approximately $10-20 \mathrm{~A} / \mathrm{m}$ known for this material. A reason for the difference in the coercive field may be that the induced stresses from cutting the samples into these dimensions, degraded considerably the crystal structure of the materials. Differences of the measured values of $J_{p}$ with manufacturer's or literature data may be due to the cross sectional determination of the specimen, a significant source of error in very small samples.

\section{Conclusions}

A sensitive computerized ac hysteresis loop tracer was built using commercial signal digitization equipment at reasonable cost. The system is capable of measuring strips, ribbons and films and is expected to be a useful tool for the characterization of new soft magnetic materials produced in NCSR "Demokritos".

\section{Acknowledgements}

The research is co-financed by Greece and the European Union under GSRT/ARISTEIA: Project No1294.

\section{References}

1. T. O'Donnell, N. Wang, R. Meere, F. Rhen, S. Roy, D. O'Sullivan, C. O'Mathuna, JMMM, e233 (2007).

2. C. R. Sullivan, S. Prabhakaran, P. Dhagat, and Y. Sun, Trans.Magn. Soc. Japan, vol. 3, no. 4, 126, (2003).

3.G. Asti, M. Ghidini, M. Solzi, JMMM, 242-245, 973975, (2002).

4. L. Kraus, O. Chayka, Journal of Electrical Engineering, 57, 8/S, 73-76, (2006).

5.J. B. Haugdahl and D. R. Miller Rev. Sci. Instrum. 59, 480 (1988).

6.F. Fiorillo "Measurement and characterization of magnetic materials”, Book, Elsevier, (2004). 